\title{
Random matrix triality at nonzero chemical potential
}

\author{
M.A. Halasz, J.C. Osborn, and J.J.M. Verbaarschot \\ Department of Physics, SUNY, Stony Brook, New York 11794
}

(June 23, 2021)

\begin{abstract}
We introduce three universality classes of chiral random matrix ensembles with a nonzero chemical potential and real, complex or quaternion real matrix elements. In the thermodynamic limit we find that the distribution of the eigenvalues in the complex plane does not depend on the Dyson index, and is given by the solution proposed by Stephanov. For a finite number of degrees of freedom, $N$, we find an accumulation of eigenvalues on the imaginary axis for real matrices, whereas for quaternion real matrices we find a depletion of eigenvalues in this domain. This effect is of order $1 / \sqrt{N}$. In particular for the real case the resolvent shows a discontinuity of order $1 / \sqrt{N}$. These results are in agreement with lattice QCD simulations with staggered fermions and recent instanton liquid simulations both for two colors and a nonzero chemical potential.
\end{abstract}

PACS numbers: 12.38.Lg, 11.30.Qc, 11.15.Ha, 11.30.Rd

Recently, nonhermitean random matrices with eigenvalues scattered in the complex plane have received a great deal of attention in both condensed matter [1 5 ] and QCD [6.7]. In condensed matter they have been used in problems ranging from neural networks [1] to the depinning transition of vortices in disordered superconductors [4.5]. In QCD, they are relevant at nonzero chemical potential when the Dirac operator is nonhermitean. In this letter we introduce three universality classes of nonhermitean random matrices with the global symmetries of the QCD partition function. This allows us to interpret certain characteristic features of quenched Dirac spectra at nonzero chemical potential (i.e. with the fermion determinant ignored in generating the field configurations) as signatures of anti-unitary symmetries. Such signatures have been found in condensed matter applications as well [2, 3, 5].

In quenched simulations of QCD it appears that the critical chemical potential $\mu_{c}$ is proportional to the pion mass which vanishes in the chiral limit as $\sqrt{m}$ ( $m$ is the quark mass) rather than to the baryon mass which remains nonzero for $m \rightarrow 0$ [8,9]. This long-standing puzzle has been resolved in a first successful application of random matrix theory to QCD at nonzero chemical potential [6]. The explanation [10,6] is that the quenched limit is the limit $N_{f} \rightarrow 0$ of a partition function with the fermion determinant to the power $N_{f}$ replaced by its absolute value. Such partition function develops a condensate with Goldstone bosons consisting of a quark and a conjugate quark. Meanwhile, several other studies have confirmed this work [7.

The above discussion was for $N_{c}=3$. For $N_{c}=2$ the situation is different 11]. Then the Dirac operator is selfconjugate leading to a real fermion determinant. Because in this case also baryons can be Goldstone bosons, we expect a critical chemical potential of $\mu_{c} \sim \sqrt{m}$. Mathematically, the Dirac operator for $N_{c}=2$ has an additional anti-unitary symmetry. As is well-known in random matrix theory, the anti-unitary symmetries lead to three different universality classes 12,13 characterized by the so called Dyson index $\beta$ ( $\beta=1$ for real, $\beta=2$ for complex, and $\beta=4$ for quaternion real matrix elements). In this letter we study a random matrix model of the QCD partition function at nonzero chemical potential for all three values of $\beta$.

The continuum Euclidean QCD partition function for $N_{f}$ flavors with masses $m_{f}$, and chemical potential $\mu$ can be written as an average over the Yang-Mills action,

$$
Z(m, \mu)=\left\langle\prod_{f=1}^{N_{f}} \operatorname{det}\left(\gamma \cdot D+m_{f}+\mu \gamma_{0}\right)\right\rangle_{S_{Q C D}},
$$

where $\gamma \cdot D$ is the Euclidean Dirac operator and $\gamma_{\mu}$ are Euclidean Dirac matrices. In lattice QCD, the chemical potential is incorporated by including a factor $e^{\mu}$ in the forward time links and a factor $e^{-\mu}$ in the backward time links. This implementation does not affect the symmetry relations discussed below.

To obtain a random matrix theory corresponding to (11) we first write the Dirac operator in a chiral basis. Then the $\mu$ independent nonzero matrix elements are replaced by Gaussian distributed random variables corresponding to the anti-unitary symmetries of the QCD partition function. In analogy with the random matrix partition function at zero [17] and nonzero temperature [14], for $N_{f}$ flavors, this partition function is defined as (see [15] for a review)

$$
Z(m, \mu)=\int D C P(C) \prod_{f}^{N_{f}} \operatorname{det}\left(D(\mu)+m_{f}\right) .
$$

Here, $C$ is an arbitrary $N \times N$ matrix with real, complex or quaternion real matrix elements. The integration measure $D C$ is the Haar measure. The probability distribution $P(C)$ is given by

$$
P(C)=\exp \left(-N(\beta / 2) \Sigma^{2} \operatorname{Tr} C C^{\dagger}\right) .
$$


From now on we will work in units where $\Sigma=1$.

In the case of $N_{c} \geq 3(\beta=2)$ there are no anti-unitary symmetries and the Dirac operator is given by [6]

$$
D(\mu)=\left(\begin{array}{cc}
0 & i C+\mu \\
i C^{\dagger}+\mu & 0
\end{array}\right) .
$$

Here, and in $(6,8)$ below, $\mu$ is proportional to the identity.

For $N_{c}=2$, the Dirac operator in (11) is subject to an additional anti-unitary symmetry [13]. We stress that a nonzero chemical potential does not violate this symmetry. For continuum fermions (and naive lattice fermions [16]) in the fundamental representation we have

$$
\left[C \tau_{2} K, i \gamma D(\mu)\right]=0,
$$

where $C$ is the charge conjugation matrix, and $i \gamma D(\mu)$ is the continuum Dirac operator at $\mu \neq 0$. Because $\left(C \tau_{2} K\right)^{2}=1$, it is possible to find a basis in which the Dirac operator is real. If we notice that $\mu \gamma_{0}$ is Hermitean we arrive at the following random matrix Dirac operator

$$
D(\mu)=\left(\begin{array}{cc}
0 & C+\mu \\
-C^{T}+\mu & 0
\end{array}\right),
$$

where $C$ is an arbitrary real matrix. Quenched instanton simulations at $\mu \neq 0$ are in this class [18].

For staggered fermions the gamma matrices are absent and for $N_{c}=2$ the anti-unitary symmetry is given by [19],

$$
\left[\tau_{2} K, D^{S}(\mu)\right]=0,
$$

where $D^{S}(\mu)$ is the staggered Dirac operator at $\mu \neq 0$. Because $\left(\tau_{2} K\right)^{2}=-1$, we can organize the matrix elements of the Dirac operator into real quaternions. For an (anti-)Hermitean Dirac operator the anti-unitary symmetry results in a pairwise degeneracy of the eigenvalues. This degeneracy is broken at $\mu \neq 0$. Then the eigenvalues of $D^{S}$ occur in complex conjugate pairs. In this symmetry class with $\beta=4$ the random matrix model is given by

$$
D(\mu)=\left(\begin{array}{cc}
0 & C+\mu \mathbf{1} \\
-C^{\dagger}+\mu \mathbf{1} & 0
\end{array}\right),
$$

where the matrix elements of $C$ are real quaternions and $\mathbf{1}$ is the unit quaternion. In addition to $D^{S}$ for $N_{c}=2$, this class also contains the Dirac operator for gauge fields in the adjoint representation 13 for $N_{c} \geq 2$.

In all three cases the spectral density at $\mu=0$ is a semicircle which in the normalization defined by (3) (with $\Sigma=1$ ) is given by

$$
\rho_{\mathrm{SC}}(\lambda)=\frac{1}{2 \pi} \sqrt{4-\lambda^{2}} .
$$

The above random matrix models apply to universality classes in which the eigenfunctions at $\mu=0$ are extended.
The eigenvalues corresponding to localized wave functions are statistically independent with spectral correlations given by the Poisson ensemble. A random matrix model in this class without a chiral structure was recently considered in [4.5]. At $\mu \neq 0$, it was found that eigenfunctions with complex eigenvalues are extended whereas eigenfunctions with real eigenvalues remain localized as for $\mu=0$. The surprising result of this study was that a localization transition was observed in one dimension. In the present context the extended states can be interpreted as the emergence of a nonzero baryon number density when the eigenvalues scatter in the complex plane at $\mu \neq 0$. Another remarkable result of this study [4] was that in the thermodynamic limit a finite number of eigenvalues remained on the real axis for $\mu$ below a critical value. Below we will show that the fraction of purely imaginary eigenvalues of the model (6) with $\beta=1$ scales as $\sim 1 / \sqrt{N}$ (Our convention and the conventions of [4: [5] for the eigenvalues differ by a factor $i$.)

However, spectral correlations of the lattice QCD Dirac operator in four dimensions are given by the invariant random matrix ensembles [20] even for relatively weak coupling. Moreover, explicit calculations of the inverse participation ratio in an instanton liquid model shows delocalized Dirac eigenfunctions [21]. Therefore, it seems that the models in which the states are localized at $\mu=0$ are inappropriate for QCD applications.

In the remainder of this paper we study the spectrum of the random matrix model (2). The generating function is given by

$$
Z=\left\langle\operatorname{det}^{n}(z-D) \operatorname{det}^{n}\left(z^{*}-D^{\dagger}\right)\right\rangle .
$$

The resolvent is defined as $G(z)=-\partial_{z} \log Z / n$ and the spectral density in the complex plane is given by $\rho(x, y)=\partial_{z^{*}} G(z) / \pi$. The quenched approximation is obtained as the limit $n \rightarrow 0$ at the end of the calculation. For integer $n$, this partition function can be analyzed in standard fashion [22,6]. First we write the determinants as Grassmann integrals. Then we perform the average over the random matrices resulting in a 4 -fermion interaction. If we bosonize this interaction and integrate out the fermions we arrive at a partition function that is amenable to a saddle point approximation. At $\mu \neq 0$ this analysis was performed by Stephanov for Hermitean random matrices. The analysis for $\beta=1$ and $\beta=4$ is somewhat more complicated 22]. However, in both cases we succeeded to solve the saddle-point equations with the remarkable result that for the normalization defined by (3), the solution coincides with the one obtained for $\beta=2$. For all values of $\beta$, the resolvent can be evaluated for arbitrary integer values of $n$. To leading order in $1 / N$ we find the result is independent of $n$. Because of the absolute value of the determinant in (10) the generating function is a smooth function of $n$. This guarantees the existence of the replica limit. 

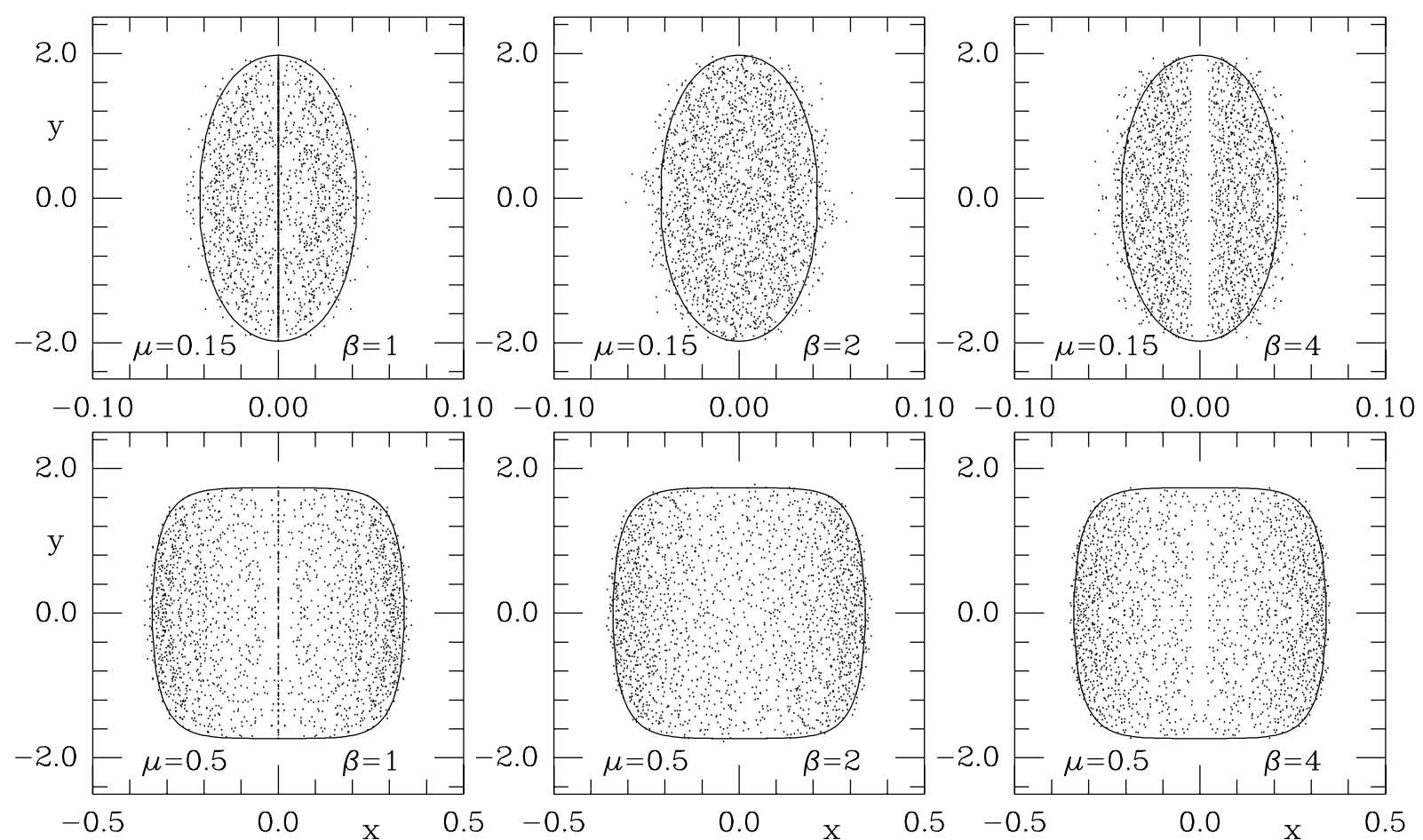

FIG. 1. Scatter plot of the real $(x)$, and the imaginary parts $(y)$ of the eigenvalues of the random matrix Dirac operator. The values of $\beta$ and $\mu$ are given in the labels of the figure. The full curve shows the analytical result for the boundary.

Numerical results for the random matrix ensembles defined in (1, 6,8) are shown in Fig. 1. We show results for $\mu=0.15$ and $\mu=0.5$. The dots represent the eigenvalues in the complex plane. The full line is the analytical result [6] for the boundary of the eigenvalues given by the algebraic curve $\left(y^{2}+4 \mu^{2}\right)\left(\mu^{2}-x^{2}\right)^{2}+x^{2}=4 \mu^{2}\left(\mu^{2}-x^{2}\right)$. For $\beta=1$ and $\beta=4$ we observe that the spectral density deviates significantly from the saddle-point result. For $\beta=1$ we find an accumulation of eigenvalues on the imaginary axis whereas for $\beta=4$ we find a depletion of eigenvalues in this domain. This depletion can be understood as follows. For $\mu=0$ all eigenvalues are doubly degenerate. This degeneracy is broken at $\mu \neq 0$ which produces the observed repulsion of the eigenvalues.

The number of purely imaginary eigenvalues appears to scale as $\sqrt{N}$, which explains that this effect is not visible in a leading order saddle point analysis. From a perturbative analysis of 10 one obtains a power series in $1 / N$. Clearly, the $\sqrt{N}$ dependence requires a truly nonperturbative analysis of (2). Such a $\sqrt{N}$ scaling behavior is typical for the regime of weak non-hermiticity first identified by Fyodorov et al. 3 .

A similar cut below a cloud of eigenvalues was found in instanton liquid simulations for $N_{c}=2$ at $\mu \neq 0$ 18 and in a random matrix model of arbitrary real matrices [2]. The depletion of the eigenvalues along the imaginary axis was observed earlier in lattice QCD simulations with staggered fermions 23].

In Fig. 2 we plot the fraction of imaginary eigenvalues versus $\mu \sqrt{N}$ for $\mu$ equal to $0.05,0.1$ and 0.2 and $N$ rang-

ing from ten to one thousand. The full line represents the analytical result due to Efetov [5]

$\alpha_{0}=\int_{-2}^{2} \rho_{S C}(\lambda) d \lambda \int_{0}^{1} d t \exp \left[-N\left(2 \pi \mu \rho_{S C}(\lambda)\right)^{2} t^{2}\right]$,

where $\rho_{S C}$ is defined in (9). This result was obtained for an ensemble of independent real symmetric matrices perturbed by an anti-symmetric matrix in the limit that the norm of the perturbing operator is of the order of the level spacing of the unperturbed matrix (his conventions differ from ours by a factor $i$ ). Apparently, the fraction of purely imaginary eigenvalues is not modified by the chiral structure of the ensemble (6). Asymptotically, for $\mu \sqrt{N} \gg 1$, this fraction is given by $\alpha_{0} \sim 1 / \mu \sqrt{\pi N}$.

It is well known that the replica trick fails in some cases [24]. For example, it fails for the unquenched partition function [6]. More typically, it fails in cases where the the saddle-point is given by a nontrivial manifold [24]. In the limit $\mu \rightarrow 0$ and $z$ real, the partition function has a higher degree of symmetry. Therefore, in the limit $N \rightarrow \infty$ with $\mu^{2} N$ fixed, the solution of the saddle point equations is given by a nontrivial manifold, and there is no guarantee that the replica trick will work. In order to obtain truly nonperturbative results one has to rely on the supersymmetric method for random matrix theory 25]. This method was extended to non-hermitean complex matrices in [26,3] and to arbitrary real matrices in [5]. The application of the supersymmetric method to the chiral ensembles will eventually provide us with an explanation of the scaling behavior of the number of purely imaginary eigenvalues ( 27]). 


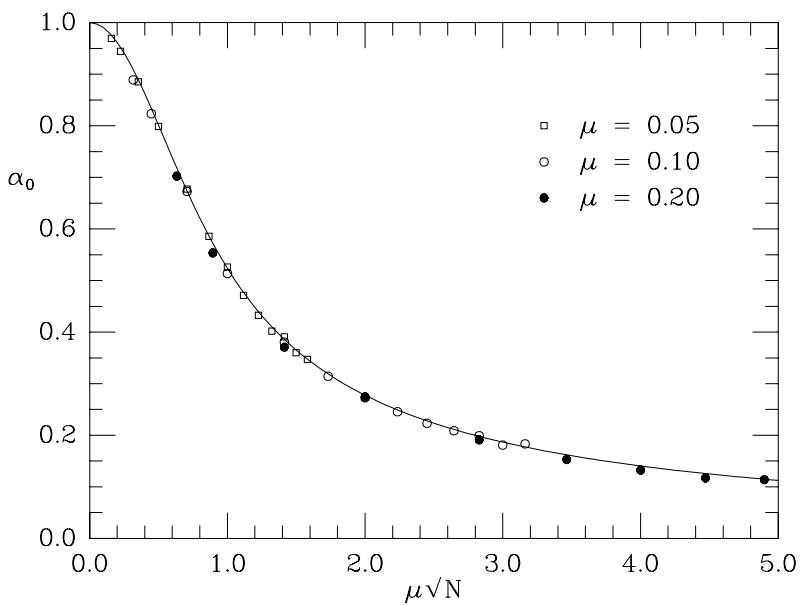

FIG. 2. The fraction of real eigenvalues, $\alpha_{0}$ versus $\mu \sqrt{N}$ for three different values of $\mu$ and matrices ranging from $N=10$ to $N=1000$. The full line shows Efetov's result $[12]$.

Stephanov has shown analytically that the quenched approximation does not work for $\beta=2$. Specifically, the unquenched partition function (2) for $N_{f} \neq 0$ results in a nonzero chiral condensate below a critical value of $\mu$. However in the quenched case, given by the $n \rightarrow 0$ limit of 10 , the chiral condensate is zero at any $\mu \neq 0$. The situation for $\beta=1$ and $\beta=4$ is different. Then in both cases the fermion determinant is real for real $z$. (For $\beta=4$ this follows by using the identity $q^{*}=\sigma_{2} q \sigma_{2}$ for a quaternion real element $q$.) Therefore, for real $z$, the generating function (10) for $n$ replicas is identical to the partition function (2) for $2 n$ flavors with mass $z$. As mentioned above, the saddle point result for the resolvent defined by the generating function (10) does not depend on $n$ and gives the quenched result for $n \rightarrow 0$. We thus conclude that quenching works for an even number of flavors. Consequently, chiral symmetry will be restored for arbitrarily small nonzero $\mu$, whereas a condensate of a quark and a conjugate quark develops. Indeed, this phenomenon has been observed in the strong coupling limit of lattice QCD with two colors [19,28].

In conclusion, at $\mu \neq 0$ we have found that the depletion of Dirac eigenvalues on the imaginary axis observed in lattice QCD simulations and the accumulation of Dirac eigenvalues found in instanton liquid simulations (both for two colors and quenched) is a generic feature related to the anti-unitary symmetries of the Dirac operator. If it turns out that the Dirac eigenfunctions are localized at $\mu=0$ (see 29] for lattice results in this direction) the fraction of purely imaginary eigenvalues at $\mu \neq 0$ might remain finite in the thermodynamic limit. For two colors, this might lead to unexpected chiral properties in the continuum limit at $\mu \neq 0$. Clearly, this possibility deserves further attention.

This work was partially supported by the US DOE grant DE-FG-88ER40388. Edward Shuryak and Yan Fy- odorov are thanked for useful discussions.

[1] H.J. Sommers, Phys. Rev. Lett. 60, 1895 (1988).

[2] B. Khoruzhenko, J. Phys. A: Math. Gen. 29, L165 (1996).

[3] Y. Fyodorov, B. Khoruzhenko and H. Sommers, Phys. Lett. A 226, 46 (1997); cond-mat/9703152.

[4] N. Hatano and D.R. Nelson, Phys. Rev. Lett. 77, 570 (1996).

[5] K.B. Efetov, cond-mat/9702091.

[6] M. Stephanov, Phys. Rev. Lett. 76, 4472 (1996).

[7] R. Janik, M. Nowak, G. Papp and I. Zahed, Phys. Rev. Lett. 77, 4816 (1996); cond-mat/9612240; J. Feinberg and A. Zee, cond-mat/9703087; M. Halasz, A. Jackson and J. Verbaarschot, Phys. Lett. B395, 293 (1997); heplat/9703006.

[8] I. Barbour et al., Nucl. Phys. B275, 296 (1986); M.P. Lombardo, J. Kogut, and D. Sinclair, hep-lat/9511026.

[9] W. Wilcox, S. Trendafilov and E. Mendel, Nucl. Phys. Proc. Supple 42 (1995) 557.

[10] A. Gocksch, Phys. Rev. Lett. 61 (1988) 2054.

[11] J. Kogut, el al., Nucl. Phys. B225 [FS9], 93 (1983).

[12] F.J. Dyson, J. Math. Phys. 3, 140 (1962).

[13] J. Verbaarschot, Phys. Rev. Lett. 72, 2531 (1994).

[14] A.D. Jackson and J. Verbaarschot, Phys. Rev. D53, 7223 (1996); T. Wettig, A. Schäfer and H. Weidenmüller, Phys. Lett. B367, 28 (1996).

[15] J. Verbaarschot, Nucl. Phys. Proc. Suppl. 53, 88 (1997).

[16] For Wilson fermions the anti-unitary symmetry is $\left[\gamma_{5} C \tau_{2} K, \gamma_{5} \gamma D^{W}\right]=0$, but the $r$-term violates the chiral symmetry, $\left\{\gamma_{5}, \gamma_{5} \gamma D^{W}\right\} \neq 0$ (see [20]).

[17] E. Shuryak and J. Verbaarschot, Nucl. Phys. A560, 306 (1993).

[18] E. Shuryak and Th. Schäfer, private communication.

[19] S. Hands and M. Teper, Nucl. Phys. B347, 819 (1990).

[20] M. Halasz and J. Verbaarschot, Phys. Rev. Lett. 74, 3920 (1995); M. Halasz, T. Kalkreuter, and J. Verbaarschot, Nucl. Phys. Proc. Suppl. 53, 266 (1997).

[21] J.C. Osborn and J. Verbaarschot, in preparation.

[22] M.A. Halasz and J. Verbaarschot, Phys. Rev. D52, 2563 (1995).

[23] C. Baillie, K. Bowler, P. Gibbs, I. Barbour and M. Rafique, Phys. Lett. 197B, 195 (1987).

[24] J. Verbaarschot and M. Zirnbauer, J. Phys. A17, 1093 (1985).

[25] K.Efetov, Adv. Phys. 32, 53 (1983); J. Verbaarschot, H. Weidenmüller, and M. Zirnbauer, Phys. Rep. 129, 367 (1985).

[26] Y. Fyodorov and H. Sommers, JETP Lett. 63 (1996), 1026.

[27] M.A. Halasz, et al., in preparation.

[28] E. Dagotto, F. Karsch and A. Moreo, Phys. Lett. 169 B, 421 (1986).

[29] K. Jansen, C. Liu, H. Simma and D. Smith, Nucl. Phys. Proc. Supp. 53, 262 (1997). 